\title{
Multi-Agent Systems Society for Power and Energy Systems Simulation
}

\author{
Gabriel Santos ${ }^{1}$, Tiago Pinto ${ }^{1,2}$, Zita Vale $^{1}$ \\ ${ }^{1}$ GECAD - Research Group on Intelligent Engineering and Computing for Advanced Innova- \\ tion and Development, Institute of Engineering, Polytechnic of Porto (ISEP/IPP), Portugal \\ \{gajls, tmcfp, zav\}@isep.ipp.pt \\ ${ }^{2}$ BISITE research group - University of Salamanca, Spain \\ tpinto@usal.es
}

\begin{abstract}
A key challenge in the power and energy field is the development of decision-support systems that enable studying problems as a whole. The interoperability between multi-agent systems that address specific parts of the global problem is essential. Ontologies ease the interoperability between heterogeneous systems providing semantic meaning to the information exchanged between the various parties. The use of ontologies within Smart Grids has been proposed describing the basic concepts used in electricity transportation and distribution. However, these ontologies are focused on the needs from largesized players and operators. The development of ontologies that allow the representation of diverse knowledge sources is essential, aiming at supporting the interaction between systems of different natures, facilitating the interoperability between these systems. This paper proposes a set of ontologies to enable the interoperability between different types of agent-based simulators, namely regarding electricity markets, the smart grid, and residential energy management. A case study based on real data shows the advantages of the proposed approach in enabling comprehensive power system simulation studies.
\end{abstract}

Keywords: Multi-agent Simulation, Power and Energy Systems, Semantic interoperability

\section{Introduction}

The emergence of liberalized electricity markets (EM) completely revolutionized the power sector business. Several challenges have been introduced during the sector's restructuring process. It required the transformation of the conceptual models that previously dominated the power sector [1].

This work has received funding from the European Union's Horizon 2020 research and innovation programme under the Marie Sklodowska-Curie grant agreement No 641794 (project DREAM-GO) and grant agreement No 703689 (project ADAPT). This work has also been supported by National funds by FCT in the scope of Gabriel Santos PhD (SFRH/BD/118487/2016) 
The market became more competitive, but also more complex, posing new challenges to its participants, forcing them to rethink their market strategies and consequently their behaviour. The new challenges that EM restructuring produced increased the importance of EM operation study. The raised complexity and competitiveness of the market together with its unpredictable evolution, hardens the decisionmaking process [2].

Several models have emerged trying to overcome market challenges. Despite the guidance provided by some pioneer countries experience in what regards the implemented market models' performance, it is still premature to take definitive conclusions. Thereby, the use of tools that allow the study of different market mechanisms and the relationships between market entities becomes essential. The use of simulation tools becomes decisive in order to study, analyse, and test different alternatives for markets' structure and evolution, providing entities with decision support tools to address the new challenges. These tools aim to deal with EM constant evolving reality, ensuring the appropriate solutions to actors adapt themselves to this new reality, acquiring experience to operate in this changing economic, financial, and regulatory environment. Operators must guarantee a transparent and competitive market while market players try to minimize their costs and maximize their profits [3].

For the analysis of dynamic and complex environments, such as the EM, multiagent based simulators are particularly well suited [4]. Multi-agent approaches enable an easy inclusion of: new market models and mechanisms, new types of market players and interactions [4]. Some reference modelling tools of this domain are: the Agent-based Modelling of Electricity Systems (AMES) [5]; the Electricity Market Complex Adaptive System (EMCAS) [6], the Multi-Agent Simulator of Competitive Electricity Markets (MASCEM) [7] and Multi-Agent Smart Grid simulation Platform (MASGriP) [8].

These are relevant tools but are often directed to the perspective of market operators and regulators, while not providing an adequate decision support to the participating players. In this scope, AiD-EM (Adaptive Decision Support for Electricity Market Negotiations) has been developed [9] with the objective of supporting players in their negotiation process.

The adequate applicability of multi-agent simulation to the study of EM has already been confirmed. However, there is a lack of interoperability between the various systems. These tools focus only on the study of the different market players and mechanisms, and on the analysis of the relations between those entities, but they still do not allow interoperability with heterogeneous tools of the same domain. These systems can benefit significantly from sharing their models with each other, allowing agent-based players from external systems to participate in the same market environment, from which they can learn from each other. Such interoperability would allow a strong improvement in EM studies and development EM simulators must be flexible in order to handle this complex and evolving reality, providing players with proper tools to adapt themselves to this dynamic reality and learn from experience. This is discussed in [10], where a short motivation for the need of developing ontologies as a way to enable the interoperability among heterogeneous Multi-Agent Systems (MAS) is addressed, culminating on the discussion on the expected advantages of creating a 
so-called Multi-Agent Systems Society, in which different systems interact with each other using the ontologies as communication language, and also enabling the interaction with other external systems that may arise in the future.

This paper provides a step forward in this domain, by introducing a set of ontologies, including the Electricity Markets Ontology (EMO) and specific ontologies for the power and energy systems' domain, designed to provide the means to achieve interoperability between power and energy multi-agent simulation platforms. Using these ontologies, a society of multi-agent systems is designed and developed. This MAS society allows integrating the various MAS, which enables the simulation of scenarios involving entities from the final consumer, through the management of resources by aggregators, to participants and operators of wholesale EM. In this way it is possible to undertake joint simulations between the various systems, allowing a more comprehensive study; and to enable the joint management of multiple knowledge sources from different natures, by integrating the systems with the several sources of data, through a common language.

After this introductory section, an overview of the proposed MAS society is provided in section 2. Section 3 presents the proposed ontologies, while section 4 features a case study based on real data from several European EM operators. Finally, the conclusions are presented in section 5 .

\section{Multi-Agent Systems Society}

Power and energy systems are complex and dynamic environments, characterized by constant changes. Studying such complex systems requires complex modelling and simulation tools, to enable capturing the complete reality. For this purpose, this work proposes a MAS architecture that is composed by multiple independent MAS, directed to the study of specific parts of the system, which, through the interaction of the involved agents, enable modelling the system as a whole.

The different MAS that compose the MAS society are developed in JAVA language and use the JADE platform to implement the agents, making the whole system FIPA (Foundation for Intelligent Physical Agents) compliant. FIPA defines a set of guidelines to reach interoperability between agent-based systems. In order to achieve the required interoperability, it is essential that agents and MAS share a common vocabulary and means of communication. In order to accomplish these requirements, agents the different MAS in this work use ontologies, which allow the sharing of vocabulary and mapping of concepts between systems, so that they can communicate. The ontologies are formulated in OWL DL, with representation in RDF/XML and are presented in section 3. In order to allow the interoperability between the systems, ontologies enable them to speak the same language and to understand the same concepts and terms, preventing different interpretations of the same information. Two types of ontologies are used. The first type is conceptual ontologies, which are the basis for communication between systems. These ontologies allow the description of the vocabulary that is shared between the systems. The second type of ontology is related to the procedural part of the systems (application ontology), and it is used to 
describe the way the systems work through the description of its services and communications, detailing inputs and outputs.

The MAS society includes several independent MAS, which cover the entire energy system, from the simulation from wholesale electricity markets until the environment inside consumers' houses. The electricity market simulation is performed by MASCEM [4]. MASCEM accommodates the simulation of a diversity of market models through a multi-agent model that includes agents to represent the market operator, the system operator, buyers, sellers and aggregators. MASCEM also enables the participation of external agents in market simulations, such as small players that are part of other systems, e.g. Smart Grid (SG) operators or other aggregators.

The decision support to market negotiations is provided by another MAS, AiD-EM [9]. AiD-EM includes agents to perform several tasks, such as the optimization of markets participation portfolio, and the decision support in auction based markets and in bilateral contracts.

The modelling of smaller players at the microgrid and SG level is provided by MASGriP, which simulates, manages and controls the most relevant players acting in a SG environment [8]. This system includes fully simulated players, which interact with software agents that control real hardware. This enables the development of a complex system capable of performing simulations with an agent society that contains both real infrastructures and simulated players, providing the means to test alternative approaches (Energy Resource Management (ERM) algorithms, Demand Response (DR), negotiation procedures, among others) in a realistic simulation setting [11].

The Intelligent Decision Support (IDeS) MAS provides several services to external systems, namely: forecast algorithms (i.e. artificial neural networks, SVM and fuzzy inference systems) to be used to forecast consumption, generation, market prices, etc; DR programs; ERM systems for SG and microgrid levels, among others. SCADA House Intelligent Management (SHIM) is a building energy management system, whose main goal is testing, simulating, and validating new algorithms and methodologies to apply in house/buildings' management [16]. In order to obtain a realistic simulation, the platform comprises real equipment such as several types of loads, mini and micro distributed generation (photovoltaic panels, wind generator), and storage systems that allow the simulation of the electric vehicles behaviour.

To ensure the simulation of complex scenarios, SHIM is able to control real loads and virtual loads simulating the characteristics of the real ones. The system is composed of different modules that are grouped into three different parts: the Data acquisition, the Actuators, and the Intelligent Applications where is included the learning algorithms. The detailed information of the structure can be found in [12]. The control of physical devices is accomplished by the connection to another MAS, the PLCMAS. The PLCMAS allows to test the scenarios in a real environment, being able to apply the results to physical devices, making them act accordingly. These devices are essentially lights, sockets and HVAC, and need to be connected to a Programmable Logic Controller (PLC).

Finally, an innovative tool is also used for the control and simulation of the MAS society. The Tools Control Centre (TOOCC) allows the simulation of the various systems/algorithms independently, as well as the joint simulation of some or all sys- 
tems present in the agent society. TOOCC also facilitates the automatic analysis of the various simulations and knowledge sources, in an integrated manner [13]. Fig. 1 shows the overall connection between the different systems.

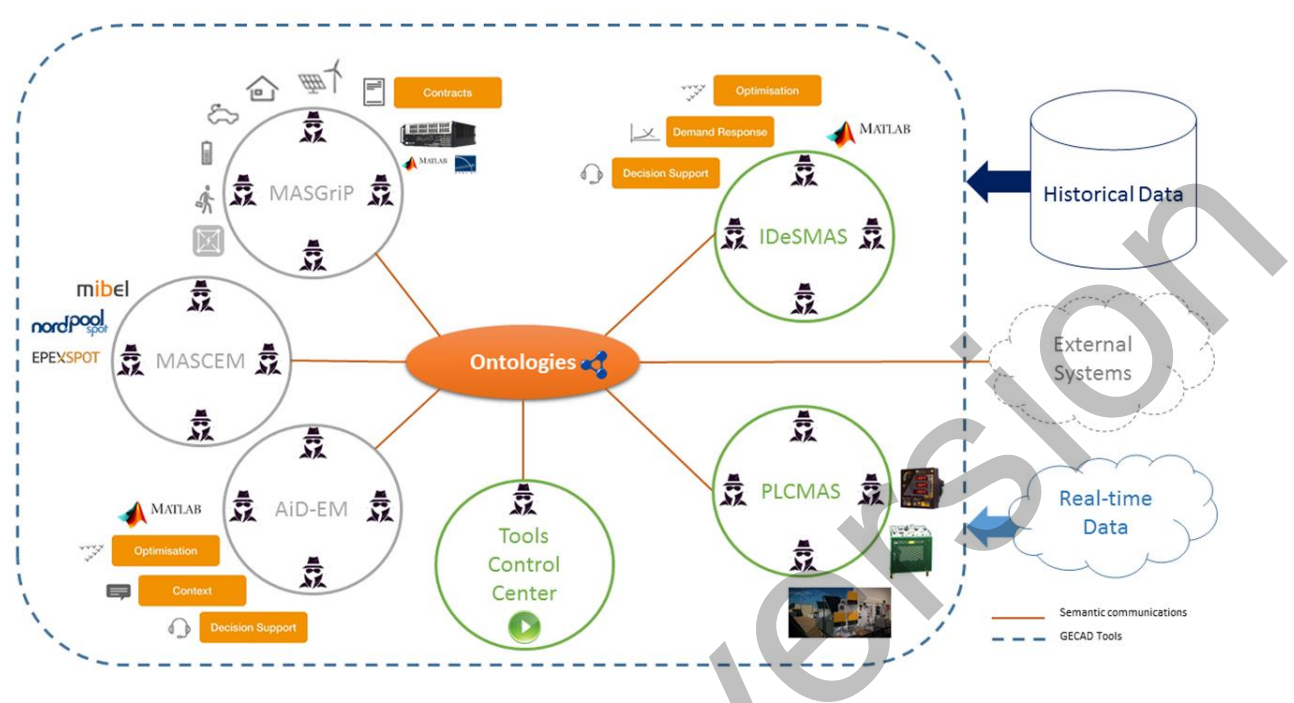

Fig. 1. Multi-Agent systems society [14]

The main advantage of the proposed MAS society is to enable the study and simulation of diverse and complex scenarios involving one or more systems devoted to distinct problems. Therefore, different complex dynamics between the agents of the different MAS can be accomplished and personalized, configured and analysed using TOOCC. The proposed MAS society enables modelling the power and energy system as a whole, by representing the most relevant players through software agents, in the respective specific MAS.

\section{Ontologies for MAS interoperability}

Currently, MAS in the power system's domain are developed with their own specific ontologies. These systems share common concepts that are differently represented between the independently developed ontologies, and translating these concepts automatically is not straightforward. In order to take full advantage of the functionalities of those systems, there is a growing need for knowledge exchange between them.

This paper proposes the use of ontologies for the interoperability of multi-agent simulation platforms in the power and energy domain, which can be extended in a way to enable the full interoperability between those systems. The ontologies provide the means to successfully exchange meaningful information that can be easily interpreted by software agents. 


\subsection{EMO specification}

The EMO incorporates abstract concepts and axioms referring to the main existing EM. This ontology aims to be as comprehensive as possible so that it can be extended and reused in the development of (lower level) market-specific ontologies. It was kept as simple as possible in order to facilitate its reuse and extension independently of the market's features and/or rules. However, given that the suggested ontologies were developed considering its use by agent based simulation tools, some markets' constraints were also defined in EMO. EMO has expressivity $A L C H I Q(D)$ [15]. Fig. 2 illustrates EMO's relations between the identified classes and object and data properties. From Fig. 2 it is possible to see the object properties represented in blue and the data properties defined within each class with the respective data types. The orange relations represent the inferred object properties, which are inverse properties of the ones defined in blue in the opposite direction.

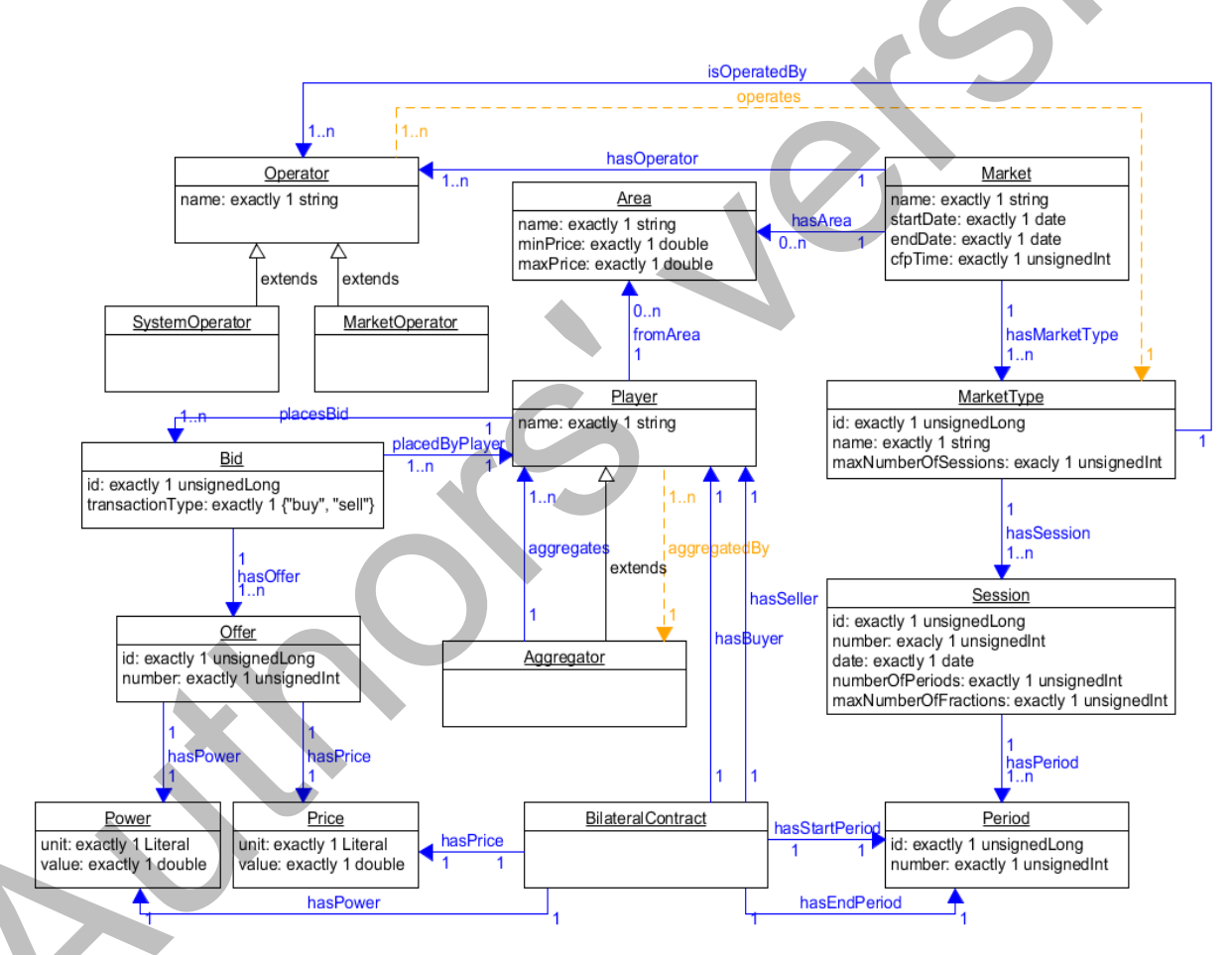

Fig. 2. Electricity Markets Ontology (EMO) ${ }^{2}$

From the concepts shown in Fig. 2, the definition of an Area includes a string name, a double minPrice and a double maxPrice. All the three data properties are defined as Functional. A functional property is a property that only relates the same

2 Available online: http://www.mascem.gecad.isep.ipp.pt/ontologies/imgs/fig2.png 
subject to one single object/value. Each EM area has an identifying name and its minimum and maximum prices are usually defined in its market rules.

An Operator includes only a name, while the MarketOperator and SystemOperator classes are extended from Operator. Other types of operators may be present in different EM, which can be defined is each market's ontology after importing the EMO.

A Period is here identified only with an id and (period) number. These two properties are both Functional as well, and it has been found important to include them in this ontology due to simulation and data storage purposes. It is certain that a period (of time) can also be defined with a start and end instants, but that terminology was left open so that, if required, one can always extend its definition in the ontology by importing EMO. Both Price and Power are defined as a set of a unit (e.g. EUR and MW respectively) and a value in double, being these two data properties Functional as well. An Offer, in turn, includes an id, a number and exactly a Power and a Price set by the object properties hasPower and hasPrice respectively. These two object properties are also Functional.

A Bid also includes an id, in addition to a transactionType ("buy" and "sell" only), a single Player (set with the Functional object property placedByPlayer) and Offers (set by the hasOffer object property). A Player includes a name, and identifies its Area and placed Bids with the respective object properties fromArea and placesBid. The placesBid object property is the inverse of placedByPlayer, being also Inverse Functional, i.e. this property only relates the same object/value to a single subject. An Aggregator, on the other hand, is a subclass of Player, which aggregates other Players. The aggregates object property is inverse of the aggregatedBy object property, being this last inferred by the reasoner when active.

A Session includes an id, a number, a date, the numberOfPeriods and the maxNumberOfFractions data properties, and also the Periods. The date data property is Functional, the numberOfPeriods identifies the number of periods to consider in the simulation, while the maxNumberOfFractions determines the maximum number of fractions (Offers) per Bid. The Periods are set with the hasPeriod object property, which is Inverse Functional. The MarketType is defined by an id, a name, the maxNumberOfSessions, including its Sessions and Operators. The maxNumberOfSessions determines the maximum number of sessions to consider in the simulation. The Sessions and Operators are set with the hasSession and isOperatedBy object properties respectively. The hasSession property is Inverse Functional and the is OperatedBy is the inverse of operates object property, which is inferred by the reasoner.

A Market comprises a name, a startDate, an endDate, a cfpTime, and its Area(s), MarketType(s) and Operator(s). The startDate, endDate and cfpTime properties are Functional. The startDate and endDate describe the simulation start and end dates, from which are also determined the number of simulation days. The cfpTime sets the call for proposal time limit a MarketOperator will wait to receive the players' proposals. The Area(s) are set through the hasArea property, the MarketType(s) by the hasMarketType property and the Operator(s) via the hasOperator object property.

A BilateralContract includes a buyer and a seller Player, a start and an end Period, a Power amount and a Price offer. The players are set by the hasBuyer and has- 
Seller Functional object properties. The start and end periods by the hasStartPeriod and hasEndPeriod properties respectively, where both are also Functional. And the hasPower and hasPrice properties set the Power and Price respectively.

Finally, the Area, the Operator, the Period, the Power, the Price, the Offer, the Player, the Bid, the Session, the Market, the MarketType and the BilateralContract classes are all Disjoint Classes, meaning that none of these classes has members in common. In other words, an element cannot be an instance of more than one of these classes, or else it makes the ontology inconsistent.

EMO was formulated in OWL DL, using Protégé $e^{3}$ tool, and its representation is in RDF/XML. It is publicly available ${ }^{4}$ so it can be used by third-party developers who wish to integrate their agent-based simulators with MASCEM, taking advantage of its simulation capabilities and market models. On the other hand, EMO may also be reused and extended for the development of new multi-agent simulation.

\subsection{Additional modules}

To enable semantic communication between the market operator and player agents, two additional modules have been developed separately from EMO. These are: (i) the Call For Proposal Ontology (CFP) and (ii) the Electricity Markets Results Ontology (EMR). Although these ontologies are not detailed in this paper, they are also publicly available ${ }^{5}$.

The CFP has the purpose of being used by the market operator agents to ask player agents for bids to be placed in the market, and for players to send their proposals to the respective market operators. In turn, the EMR is the ontology used by market operator agents to inform player agents about their results and outcomes in the market. Both modules have expressivity $\operatorname{ALCHIQ}(D)$, similarly to EMO, and have also been formulated in OWL DL, being represented in RDF/XML as well.

As in usual ontological development good practices, CFP and EMR are independent from EMO as they do not directly integrate concepts that define an electricity market; rather concepts that are related to an electricity market.

\subsection{Complementary ontologies}

The electricity market ontologies are complemented by a large number of other ontologies that are useful not only for communication purposes, but also for knowledge representation and sharing among the software agents. Most concepts represented by these ontologies are shared by multiple systems, in particular the concepts referring to smaller-sized energy resources and devices, namely the ones part of MASGriP, SHIM and PLCMAS. These ontologies can be summarized as follows:

\footnotetext{
http://protege.stanford.edu/

http://www.mascem.gecad.isep.ipp.pt/ontologies/electricity-markets.owl

http://www.mascem.gecad.isep.ipp.pt/ontologies/call-for-proposal.owl,

http://www.mascem.gecad.isep.ipp.pt/ontologies/electricity-markets-results.owl
} 
- SEAS-ActorOntology: The SEAS Actor Ontology defined for the ITEA2 SEAS project

- SEAS-ActorVocabulary: The Seas Actor Vocabulary defined for the ITEA2 SEAS project

- SEAS-AreaOntology: This ontology is targeted for defining structure of buildings (or more general facilities) and zones related to control and measurement with links to various BIM (Building Information Model) related standards

- SEAS-BuildingCategoriesVocabulary: The SEAS Building Categories for the ITEA2 SEAS (Smart Energy Aware Systems) project. This module presents subcategories for classifying building related

- SEAS-EnergyFormVocabulary: This vocabulary defines forms of energy, such as ElectricEnergy, NuclearEnergy, MagneticEnergy or ThermalEnergy

- SEAS-ElectricityPlayerOntology: This ontology defines electricity players and electricity market, as systems that exchange electricity

- SEAS-ElectricPowerSystemVocabulary: The SEAS Electric Power System Vocabulary defines: 1) Electric power systems that consume, produce, or store electricity, 2) electrical connections between electric power systems, where electricity is exchanged, and 3) electrical connection Points of electric power systems, through which electricity flows in/out the power systems

- SEAS-FlexibilityVocabulary: The SEAS Flexibility Vocabulary defines code lists to interpret evaluations of operating features of interest. For instance, the value may be the minimal operating value

- SEAS-LightSystemOntology: This vocabulary defines light Systems, and their common properties

- SEAS-ThermodynamicSystemOntology: This ontology is targeted for defining thermodynamic systems and their relations

- SEAS-ThermodynamicSystemVocabulary: This vocabulary defines common properties of thermodynamic systems, and evaluation interpretation code lists

The developed ontologies not only enable the interoperability between different MAS but also represent the concepts needed to understand and use empirical data, from different sources. These data can be acquired in real time through analysers/sensors, or even databases available online. For that, the developed ontologies allow the representation of knowledge in a common vocabulary, regardless of the source; thus facilitating interoperability between the various heterogeneous systems and data, information and knowledge sources, with the ultimate goal of achieving an enhanced simulation platform for fully transactive energy systems.

\section{Case Study}

This case study intends to demonstrate the usefulness and advantage of using EMO to support players' participation in the market. The simulation scenario was created with the intention of representing the European reality through a summarized group of players, representing buyer and seller entities of each area of each regional market. It includes two agents (buyer and seller) per area, practicing the average prices and ne- 
gotiating the total amount of power that have been transacted in each of these areas in the reality, for the day $16^{\text {th }}$ January, 2013 (Wednesday).

Forty one areas are considered, i.e. 41 buyers and 41 sellers, resulting in a total of 82 players for this simulation. The selected market type is the symmetrical day-ahead pool without considering any complex offer or condition.

As the simulation starts, the market operator sends a call for proposal (CfP) to each registered player. Fig. 3 presents a snippet of the CfP sent by the market operator. The full version can be found online ${ }^{6}$.

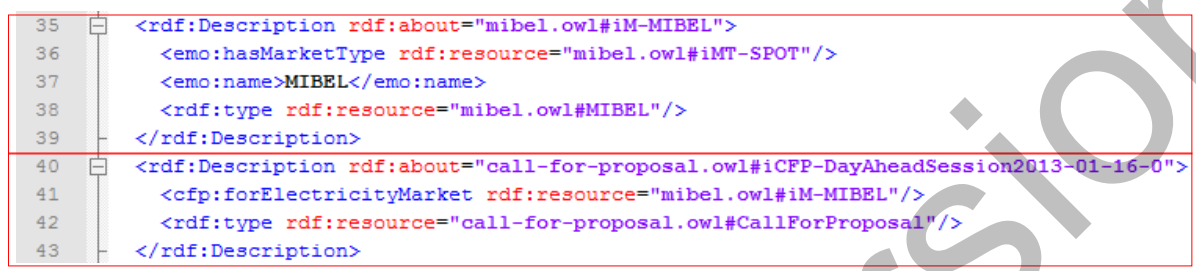

Fig. 3. CfP RDF snippet

Analyzing Fig. 3, it is possible to observe the definition of a CallForProposal (from line 40 to line 43) for the EM named "MIBEL" (defined from line 35 to 39).

After receiving the $\mathrm{CfP}$, each player queries its knowledge base in order to send its proposal to the respective market operator. Fig. 4 presents a snip of the Proposal sent by agent Seller 38. The complete version is available online ${ }^{7}$.

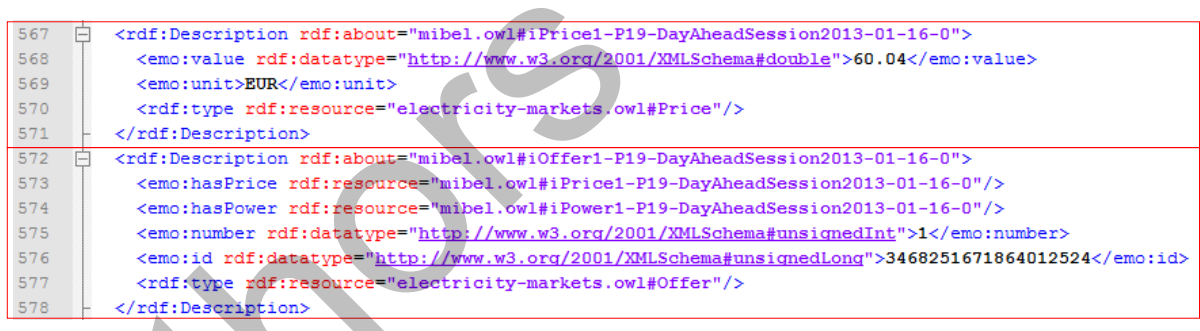

Fig. 4. Seller 38's Proposal RDF snippet

Observing Fig. 4, it is noticeable the definition of an Offer for period 19 (between lines 572 and 578). The Price proposed for this offer is defined from line 567 to 571.

After receiving the proposals and validating all incoming offers, the market operator analyses the bids, and generates the RDF results to be sent to the participating players. An excerpt of the RDF result achieved by Seller 38 is illustrated in Fig. 5. The full version of this RDF can be found online ${ }^{8}$, where the results may be observed with better insight.

\footnotetext{
6 http://www.mascem.gecad.isep.ipp.pt/ontologies/paper/paams/16/CfP.rdf

7 http://www.mascem.gecad.isep.ipp.pt/ontologies/paper/paams/16/Proposal.rdf

8 http://www.mascem.gecad.isep.ipp.pt/ontologies/paper/paams/16/Result.rdf
} 


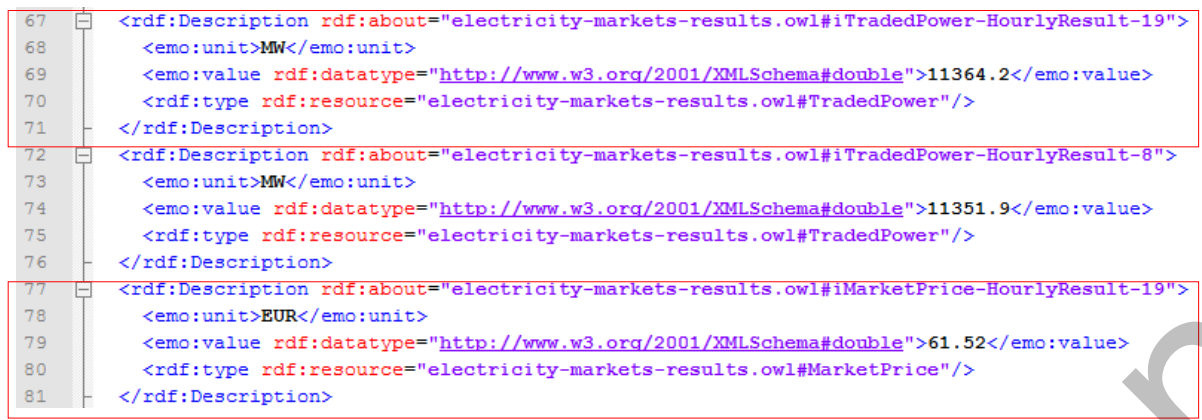

Fig. 5. Seller 38's Result RDF snippet

By the analysis of Fig. 5 it is possible to observe the traded power of Seller 38 (between lines 67 and 71 ) and market clearing price (from line 77 to line 81 ) of period 19.

Fig. 6 presents the results achieved by Seller 38 for each hourly period of the considered day. As it is possible to notice, Seller 38 sold almost all its available power for the 24 hourly periods of the day. In periods 4 and 6 Seller 38 was not able to sell any of the offered power. In turn, in period 5, this agent is the one who determines the market price (agent's bid price is equal to the market price), being only able to sell less than $1 / 4$ of its available power. Market prices vary approximately between $€ 38$ (period 4) and $€ 75$ (period 10).

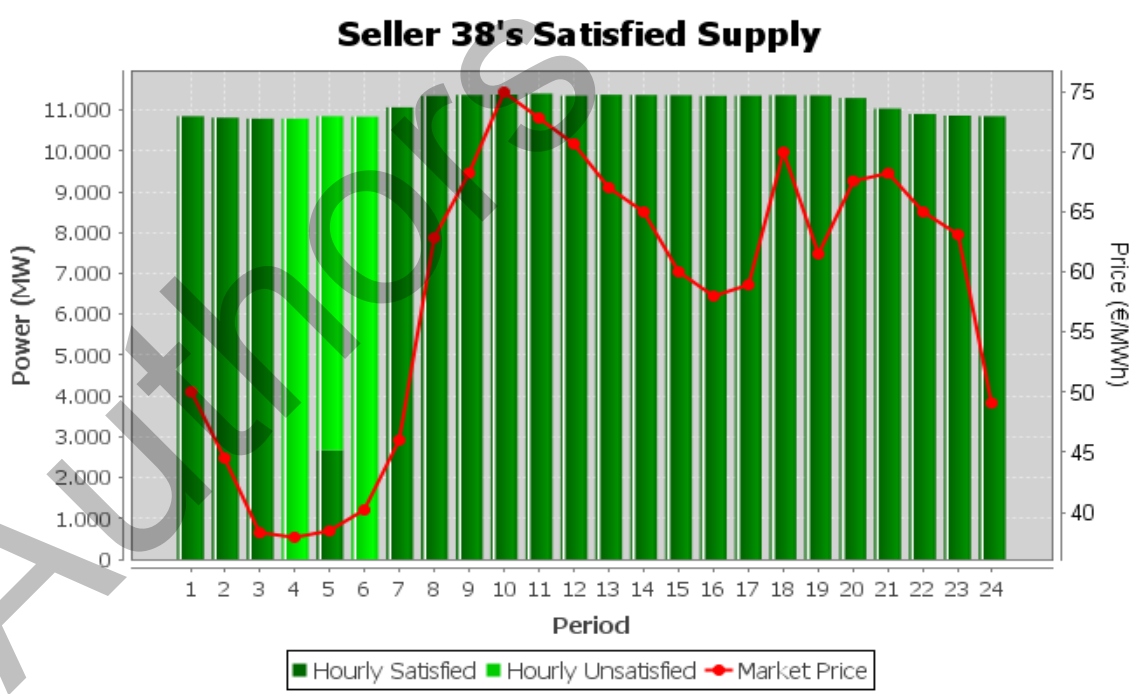

Fig. 6. Seller 38's satisfied supply 


\section{Conclusions}

In order to disseminate the development of interoperable MAS within power engineering, interconnection issues must be addressed. To take full advantage of these systems, there is a growing need for knowledge exchange with the aim at providing full interoperability between different systems. With the objective of overcoming these issues, the Electricity Markets Ontology (EMO) is proposed, gathering the EM main concepts, enabling the interoperability of independent multi-agent based simulation platforms.

Additionally, particular modules conceived to deal with the different communications between power and energy players have also been developed. Using these ontologies, different types of agents are able to communicate with each other, understanding a common language, while providing the means for any agent from external systems to do the same, simply by importing the developed ontologies. By "speaking the same language", agents from different communities can understand each other and communicate efficiently, without the need for spending unnecessary computational resources and execution time (essential in a simulation process) in translating messages.

The developed ontology is publicly available online so it can be easily accessed, reused and extended by Ontology Engineers or MAS developers in the scope of EM. This is a relevant contribution, not only to provide the participation in joint simulations in the power and energy domain, but also to give the basis for the development of other systems specific ontologies. The comparison of the system's performance with and without the use of ontologies is considered as future work, as well as the conversion of RDF messages to JSON-LD ${ }^{9}$ to reduce the computational weight of communications.

The presented case study has shown the usefulness of using the proposed ontologies in the scope of EM simulations. The new MAS society resulting from the integration of the proposed ontologies for interoperability of several MAS simulators, provides a solid platform to study and explore the implications and consequences of new and already existing approaches in EM. Researchers of the power systems area consider tools with this type of capabilities essential in order to be prepared to deal with the constant changes in the EM environment.

\section{References}

1. Sioshansi, F.P.: Evolution of global electricity markets: new paradigms, new challenges, new approaches. Elsevier Science \& Technology, Waltham, MA (2013).

2. Ciarreta, A., Espinosa, M.P., Pizarro-Irizar, C.: Has renewable energy induced competitive behavior in the Spanish electricity market? Energy Policy. 104, 171-182 (2017).

3. Geng, Z., Conejo, A.J., Chen, Q., Xia, Q., Kang, C.: Electricity production scheduling

\footnotetext{
${ }^{9}$ http://json-ld.org/
} 
under uncertainty: Max social welfare vs. min emission vs. max renewable production. Appl. Energy. 193, 540-549 (2017).

4. Santos, G., Pinto, T., Praça, I., Vale, Z.: MASCEM: Optimizing the performance of a multi-agent system. Energy. 111, 513-524 (2016).

5. Li, H., Tesfatsion, L.: Development of Open Source Software for Power Market Research: The AMES Test Bed. J. Energy Mark. 2, 111-128 (2009).

6. Thimmapuram, P., Veselka, T.D., Koritarov, V., Vilela, S., Pereira, R., Silva, R.F.: Modeling hydro power plants in deregulated electricity markets: Integration and application of EMCAS and VALORAGUA. In: 2008 5th International Conference on the European Electricity Market, EEM (2008).

7. Vale, Z., Pinto, T., Praça, I., Morais, H.: MASCEM: Electricity Markets Simulation with Strategic Agents. IEEE Intell. Syst. 26, 9-17 (2011).

8. Oliveira, P., Pinto, T., Morais, H., Vale, Z.: MASGriP a multi-agent smart grid simulation platform. IEEE Power Energy Soc. Gen. Meet. 1-8 (2012).

9. Pinto, T., Vale, Z., Sousa, T.M., Praça, I., Santos, G., Morais, H.: Adaptive learning in agents behaviour: A framework for electricity markets simulation. Integr. Comput. Aided. Eng. 21, 399-415 (2014).

10. Santos, G., Pinto, T., Vale, Z.: Ontologies for the Interoperability of Heterogeneous Multi-agent Systems in the Scope of Power and Energy Systems. In: De la Prieta, F., Vale, Z., Antunes, L., Pinto, T., Campbell, A.T., Julián, V., Neves, A.J.R., and Moreno, M.N. (eds.) BT - Trends in Cyber-Physical Multi-Agent Systems. The PAAMS Collection - 15th International Conference, PAAMS 2017. pp. 300-301. Springer International Publishing, Cham (2017).

11. Gomes, L., Faria, P., Morais, H., Vale, Z., Ramos, C.: Distributed, agent-based intelligent system for demand response program simulation in smart grids. IEEE Intell. Syst. 29, 56-65 (2014).

12. Faia, R., Pinto, T., Abrishambaf, O., Fernandes, F., Vale, Z., Corchado, J.M.: Case based reasoning with expert system and swarm intelligence to determine energy reduction in buildings energy management. Energy Build. 155, 269-281 (2017).

13. Teixeira, B., Pinto, T., Silva, F., Santos, G., Praça, I., Vale, Z.: Multi-Agent Decision Support Tool to Enable Interoperability among Heterogeneous Energy Systems. Appl. Sci. 8, (2018).

14. Pinto, T., Faia, R., Navarro-Caceres, M., Santos, G., Corchado, J.M., Vale, Z.: MultiAgent-Based CBR Recommender System for Intelligent Energy Management in Buildings. IEEE Syst. J. 1-12 (2018).

15. Borgwardt, S., Lippmann, M., Thost, V.: Temporalizing rewritable query languages over knowledge bases. J. Web Semant. 33, 50-70 (2015). 